\title{
GS-967 and Eleclazine Block Sodium Channels in Human Induced Pluripotent Stem Cell-Derived Cardiomyocytes ${ }^{\mathrm{S}}$
}

\author{
Franck Potet, Defne E. Egecioglu, Paul W. Burridge, and Alfred L. George, Jr. \\ Department of Pharmacology, Northwestern University Feinberg School of Medicine, Chicago, Illinois
}

Received April 26, 2020; accepted August 24, 2020

\section{ABSTRACT}

GS-967 and eleclazine (GS-6615) are novel sodium channel inhibitors exhibiting antiarrhythmic effects in various in vitro and in vivo models. The antiarrhythmic mechanism has been attributed to preferential suppression of late sodium current $\left(I_{\mathrm{NaL}}\right)$. Here, we took advantage of a high throughput automated electrophysiology platform (SyncroPatch 768PE) to investigate the molecular pharmacology of GS-967 and eleclazine on peak sodium current $\left(I_{\mathrm{NaP}}\right)$ recorded from human induced pluripotent stem cell-derived cardiomyocytes. We compared the effects of GS-967 and eleclazine with the antiarrhythmic drug lidocaine, the prototype $I_{\mathrm{NaL}}$ inhibitor ranolazine, and the slow inactivation enhancing drug lacosamide. In human induced pluripotent stem cell-derived cardiomyocytes, GS-967 and eleclazine caused a reduction of $/ I_{\mathrm{NaP}}$ in a frequencydependent manner consistent with use-dependent block (UDB). GS-967 and eleclazine had similar efficacy but evoked more potent UDB of $I_{\mathrm{NaP}}\left(\mathrm{IC}_{50}=0.07\right.$ and $0.6 \mu \mathrm{M}$, respectively) than ranolazine $(7.8 \mu \mathrm{M})$, lidocaine $(133.5 \mu \mathrm{M})$, and lacosamide (158.5 $\mu \mathrm{M})$. In addition, GS-967 and eleclazine exerted more potent

\section{Introduction}

Sodium current $\left(I_{\mathrm{Na}}\right)$ in cardiac myocytes carried primarily by sodium-gated voltage channel $\left(\mathrm{Na}_{\mathrm{V}}\right) 1.5$ channels is responsible for the rapid upstroke of atrial and ventricular action potentials as well as the rapid propagation of depolarization throughout the heart. Sodium channels can transition between at least three distinct states: resting (closed), open (active), and inactivated (nonconducting) (Hodgkin and Huxley, 1952). The transitions between these states are voltage and time dependent. Antiarrhythmic, anticonvulsant, and local anesthetic agents have been shown to block the propagation of action potentials by interacting differently with each channel state

Dr. Potet is a paid consultant for Praxis Precision Medicines, Inc. Dr. George serves on a scientific advisory board for Amgen, Inc. and received previous grant support from Merck and Co., Gilead Sciences, Inc., and Praxis Precision Medicines, Inc.

An earlier version of this paper appears in bioRxiv under the DOI: $10.1101 /$ 2020.05.08.084350.

This work was funded in part by a grant from Fondation Leducq and through research investments by the Northwestern Medicine Catalyst Fund.

https://doi.org/10.1124/molpharm.120.000048.

S This article has supplemental material available at molpharm. aspetjournals.org. effects on slow inactivation and recovery from inactivation compared with the other sodium channel blocking drugs we tested. The greater UDB potency of GS-967 and eleclazine was attributed to the higher association rates and moderate unbinding rate of these two compounds with sodium channels. We propose that substantial UDB contributes to the observed antiarrhythmic efficacy of GS-967 and eleclazine.

\section{SIGNIFICANCE STATEMENT}

We investigated the molecular pharmacology of GS-967 and eleclazine on sodium channels in human induced pluripotent stem cell-derived cardiomyocytes using a high throughput automated electrophysiology platform. Sodium channel inhibition by GS-967 and eleclazine has unique effects, including accelerating the onset of slow inactivation and impairing recovery from inactivation. These effects combined with rapid binding and moderate unbinding kinetics explain potent usedependent block, which we propose contributes to their observed antiarrhythmic efficacy.

ABBREVIATIONS: CDM3, chemically defined medium 3; DPBS, Dulbecco's PBS; hiPSC, human induced pluripotent stem cell; $I_{\mathrm{Na}}$, sodium current; $I_{\mathrm{NaL}}$, late sodium current; $I_{\mathrm{NaP}}$, peak sodium current; $\mathrm{K}_{\mathrm{OFF}}$, unbinding rate; $\mathrm{K}_{\mathrm{ON}}$, binding rate; $\mathrm{Na}_{\mathrm{V}}$, voltage-gated sodium channel; UDB, usedependent block. 
certain acquired or heritable conditions, including failing and/or ischemic heart (Le Grand et al., 1995), oxidative stress (Song et al., 2006), or mutations in SCN5A, which encodes Nav1.5 (Bennett et al., 1995; Ruan et al., 2009). SCN5A mutations that cause enhanced $I_{\mathrm{NaL}}$ produce type 3 long QT syndrome (Antzelevitch et al., 2014). Drugs that selectively suppress $I_{\mathrm{NaL}}$ may offer a targeted antiarrhythmic strategy in these conditions.

Many local anesthetic and antiarrhythmic agents have greater potency to block $I_{\mathrm{NaL}}$ than peak $I_{\mathrm{Na}}\left(I_{\mathrm{NaP}}\right)$. Certain compounds, such as ranolazine (Gupta et al., 2015) and F15845 (Pignier et al., 2010), are described as preferential $I_{\mathrm{NaL}}$ blockers. GS-967 [a triazolopyridine derivative, 6-(4(trifluoromethoxy)phenyl)-3-(trifluoromethyl)-[1,2,4]triazolo [4,3-a]pyridine, also referred as PRAX-330] (Koltun et al., 2016) and eleclazine (dihydrobenzoxazepinone, formerly known as GS-6615) are recently described sodium channel blockers that were originally demonstrated to exert potent antiarrhythmic effects in rabbit ventricular, canine, and pig atrial myocytes by a proposed mechanism of action involving preferential $I_{\mathrm{NaL}}$ block (Belardinelli et al., 2013; Sicouri et al., 2013; Fuller et al., 2016). We previously demonstrated that GS-967, in addition to blocking $I_{\mathrm{NaL}}$, also exerts a strong usedependent block (UDB) of $I_{\mathrm{NaP}}$ conducted by heterologously expressed recombinant human $\mathrm{Na}_{\mathrm{V}} 1.5$ and proposed that this phenomenon might contribute to its antiarrhythmic effect (Potet et al., 2016). Further studies examining the molecular pharmacology of GS-967 and eleclazine on sodium channels in cardiomyocytes should provide valuable insight into the drugs' mechanism of action in a therapeutically relevant cell type.

In this study, we investigated the molecular pharmacology of GS-967 and eleclazine in human induced pluripotent stem cell (hiPSC)-derived cardiomyocytes using a high throughput automated electrophysiology platform (SyncroPatch 768PE). We compared GS-967 and eleclazine with lidocaine (a class Ib antiarrhythmic drug that promotes UDB) (Herzog et al., 2003 ), with ranolazine (a prototype $I_{\mathrm{NaL}}$ inhibitor with class Ib antiarrhythmic characteristics) (Szél et al., 2011), and with lacosamide (an anticonvulsant drug that enhances slow inactivation) (Errington et al., 2008). Both, ranolazine and lacosamide can enhance slow inactivation of $\mathrm{Na}_{\mathrm{V}}$ channels (Errington et al., 2008; Kahlig et al., 2014). We observed that eleclazine, like GS-967, exhibits moderate dissociation rates, comparable to the class Ib antiarrhythmic lidocaine, along with uniquely rapid binding kinetics. These properties explain the potent use-dependent block of $I_{\mathrm{NaP}}$ by GS-967 and eleclazine observed in hiPSC-derived cardiomyocytes.

\section{Materials and Methods}

Cell Culture. hiPSCs were derived from peripheral blood of a male donor as previously described (Burridge et al., 2016). The resulting line was designated $113 \mathrm{c} 4$. The hiPSC line was cultured on growth factor-reduced Matrigel (Corning, NY) in E8 medium (Burridge et al., 2015). Cardiac differentiation was completed in chemically defined medium 3 (CDM3) as previously described (Burridge et al., 2014). CDM3 consisted of RPMI 1640 (Corning), $500 \mu \mathrm{g} / \mathrm{ml}$ fatty acid-free albumin (GenDEPOT, Barker, TX), and $200 \mu \mathrm{g} / \mathrm{ml} \mathrm{l-ascorbic} \mathrm{acid} \mathrm{2-}$ phosphate (Wako, Richmond, VA). At day 20 of differentiation, cells were dissociated by incubation in Dulbecco's PBS (DPBS) (without $\mathrm{Mg}^{2+}$ or $\mathrm{Ca}^{2+}$ ) for 20 minutes at $37^{\circ} \mathrm{C}$ and then in 1:200 Liberase $\mathrm{TH}$ (Roche, Basel, Switzerland) in DPBS for 20 minutes at $37^{\circ} \mathrm{C}$. Cells were collected by centrifuging at $200 \mathrm{~g}$ for 3 minutes, counted, then replated in Matrigel-coated six-well plates at 2-4 million cells per well in CDM3 supplemented with 40\% FBS (Opti-Gold; GenDEPOT, Katy, TX). Cells were returned to CDM3 on day 22. hiPSC-derived cardiomyocytes were replated in $30 \mathrm{~mm}$ culture dishes 5 days prior to the experiment.

Automated Patch Clamp Recording. Automated patch clamp recording was performed using a Syncropatch 768 PE (Nanion Technologies, Munich, Germany). On the day of the experiment, cells were washed once with DPBS (Mg/Ca free) for 20 minutes. Cells were then detached with 5-minute treatment with TrypLE followed by 20-30 minutes' treatment with CDM3 medium with 1:200 dilution of Liberase TH. Cells were then re-suspended in 15\% CDM3 medium and $85 \%$ external solution at 170,000 cells per milliliter. Cells were allowed to recover for at least 30 minutes at $15^{\circ} \mathrm{C}$ while shaking on a rotating platform. After equilibration, $10 \mu \mathrm{l}$ of cell suspension was added to each well of a 384-well, single-hole, low resistance (3 M $\Omega$ ) NPC-384 "chip" (Nanion Technologies).

Pulse generation and data collection were done with PatchController384 version 1.3.0 and DataController384 version 1.2.1 (Nanion Technologies). Whole-cell currents were filtered at $3 \mathrm{kHz}$ and acquired at $10 \mathrm{kHz}$. The access resistance and apparent membrane capacitance were estimated using protocols within the data acquisition software. Series resistance was compensated $95 \%$, and leak and capacitance artifacts were subtracted out using the P/4 method. Whole-cell currents were recorded at room temperature $\left(\sim 24^{\circ} \mathrm{C}\right)$ in the wholecell configuration. The external solution contained (in millimolars) $\mathrm{NaCl} 140, \mathrm{KCl} 4, \mathrm{CaCl}_{2} 2, \mathrm{MgCl}_{2} 1$, HEPES 10, glucose 5, $\mathrm{pH}$ 7.4. The internal solution contained (in millimolars) CsF 110, CsCl 10, NaCl 10, HEPES 10, EGTA 20, pH 7.2.

Using hiPSC-derived cardiomyocytes, the average cell catch per plate was $58 \% \pm 4 \%$ of wells (ranging from $22 \%$ to $84 \%$ ). The average number of cells per plate with a seal resistance $>0.25 \mathrm{G} \Omega$ was $48 \% \pm$ $5 \%$ (range 11\%-80\%), and among these cells, 56\% $\pm 4 \%$ (range $12 \%-91 \%$ ) expressed sodium current. Only cells with good voltage control and a peak current $\geq 300 \mathrm{pA}$ were selected for analysis. Using these criteria, the success rate of recording sodium current from hiPSC-derived cardiomyocytes was $25 \% \pm 2 \%$ of wells. The average seal resistance of the cells selected for analysis was $0.618 \pm 0.05 \mathrm{G} \Omega$, and the average capacitance was $18.3 \pm 0.7 \mathrm{pF}$. The average access resistance was $5.6 \mathrm{M} \Omega$, and the calculated voltage error was $3.2 \mathrm{mV}$.

Drugs were diluted in the external solution and prepared in a separate 24-well plate. GS-967 was obtained from Gilead (Foster City, CA). Eleclazine, lidocaine, ranolazine, and lacosamide were obtained from Sigma Aldrich (St. Louis, MO). DMSO concentration was the same for each concentration of a given drug. To block voltagegated $\mathrm{Ca}^{2+}$ channels, $0.5 \mu \mathrm{M}$ nisoldipine (Sigma Aldrich) was added to external solutions.

Data Analysis. Cells were excluded from analysis if the maximum peak current was less than $300 \mathrm{pA}$, which was a predetermined threshold. Patch-clamp measurements are presented as means \pm S.D. or S.E.M. (used for clarity in data-dense plots). Half maximal inhibitory concentration $\left(\mathrm{IC}_{50}\right.$ ) values were calculated by fitting the dose response curves with a four parameter logistic equation, where $\%_{\text {inhibition }}=\operatorname{Min} \%_{\text {inhibition }}+\left(\mathrm{Max}_{\% \text { inhibition }}-\mathrm{Min}_{\% \text { inhibition }}\right) /(1+$ $\left.\left([\mathrm{drug}] / \mathrm{IC}_{50}\right)^{-\mathrm{n}}\right)$, in which $\%_{\text {inhibition }}$ represents the percentage of peak current inhibited after block by each drug, Min \%inhibition and Max\% inhibition are the minimal and maximum \% block that best fit the plateaus of the curve for each drugs, [drug] is the concentration of drug, and $n$ is the slope. The four parameters were fit with no constraints except for the tonic block of lidocaine and lacosamide (Fig. 1, D and E) for which the Max\%inhibition was constrained to a maximum value of $100 \%$.

The apparent binding rate $\left(\mathrm{K}_{\mathrm{ON}}\right)$ was measured using the timedependent inhibition of $I_{\mathrm{Na}}$ during a variable length conditioning pulse. The time-dependent inhibition of $I_{\mathrm{NaP}}$ for each concentration was fitted with an exponential decay function having a time constant $(\tau)$. Values for $1 / \tau$ were plotted against the drug concentrations and best fitted with a linear function. The slope represents the $\mathrm{K}_{\mathrm{ON}}$ of the drug. The apparent unbinding rate $\left(\mathrm{K}_{\mathrm{OFF}}\right)$ was measured using the 
A

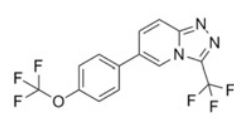

GS-967

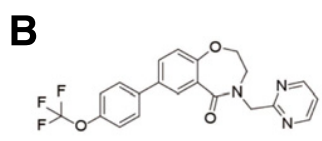

Eleclazine

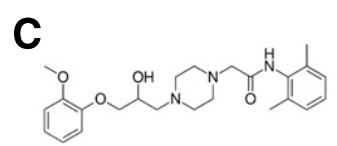

Ranolazine
D

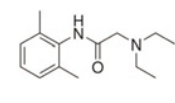

Lidocaine
E

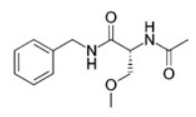

Lacosamide
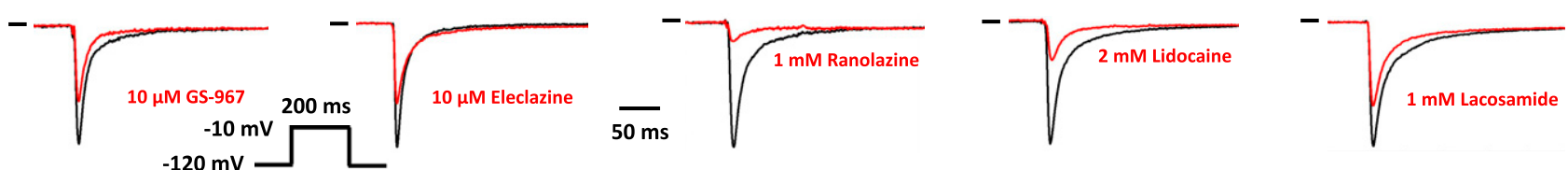

GS-967 Tonic Block

Eleclazine Tonic Block

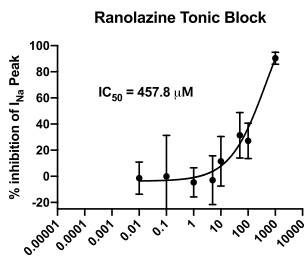

Concentration (uM)

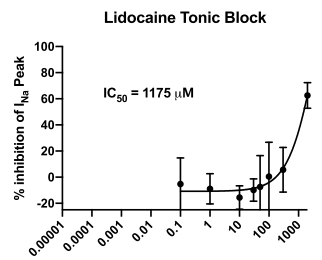

Concentration ( $\mu \mathrm{M})$

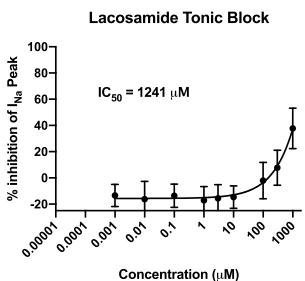

Fig. 1. $I_{\mathrm{NaP}}$ tonic block in hiPSC-derived cardiomyocytes. Top: Drug chemical structures. Middle: Representative $I_{\mathrm{NaP}}$ traces in absence (black) and presence (red) of the drug. Bottom: $I_{\mathrm{NaP}}$ concentration-response curve for GS-967 (A), eleclazine (B), ranolazine (C), lidocaine (D), and lacosamide (E). Currents were recorded from hiPSC-derived cardiomyocytes using automated patch clamp. hiPSC-cardiomyocytes were held at a membrane potential of $-120 \mathrm{mV}$ and depolarized every 5 seconds to $-10 \mathrm{mV}$ for 200 milliseconds (see inset). $I_{\mathrm{NaP}}$ was measured at $-10 \mathrm{mV}$ before drug and was measured again 5 minutes after addition of the drug. The data are shown as percentages of $I_{\mathrm{NaP}}$ normalized to the maximum current in the absence of drug $\left(I / I_{\text {max }}\right)$. The representative current traces shown for the blocking effects of GS-967, eleclazine, ranolazine, lidocaine, and lacosamide on $I_{\mathrm{NaP}} 5$ minutes after perfusion were chosen at a concentration giving the maximum tonic block. The curves described by the solid lines were fitted by a four-parameter logistic equation. Each data symbol in the concentration response curves represents mean \pm S.D. for $n=7-67$ cells. Estimated $\mathrm{IC}_{50}$ values are indicated for each concentration-response curve. The 95\% confidence intervals and Hill coefficients are provided in Table 1.

time-dependent delay in the recovery of $I_{\mathrm{Na}}$ after an inactivating conditioning pulse. The recovery time course for each drug was fit with a single exponential equation to obtain a recovery time constant, $\tau_{\mathrm{OFF}}$. The recovery time constants of the three highest concentrations used for each drug was averaged, and the $\mathrm{K}_{\mathrm{OFF}}$ was calculated using $\mathrm{K}_{\mathrm{OFF}}=1 / \tau_{\mathrm{OFF}}$

\section{Results}

Eleclazine and GS-967 Exert Limited Tonic Block in hiPSC-Derived Cardiomyocytes. We previously reported that $1 \mu \mathrm{M}$ GS-967 inhibits $18 \%$ of $I_{\mathrm{NaP}}$ conducted by human $\mathrm{Na}_{\mathrm{V}} 1.5$ channels expressed in heterologous (tsA201) cells (Potet et al., 2016). Based on this finding, we predicted that GS-967 and eleclazine would exhibit similar effects on $I_{\mathrm{NaP}}$ in hiPSC-derived cardiomyocytes. We performed automated patch clamp recordings of hiPSC-derived cardiomyocytes using the Syncropatch 768PE platform. Based on RNAsequencing data performed on our hiPSC-derived cardiomyocytes, SCN5A (Nav1.5) accounts for $84 \%$ of the expressed sodium channels (data not shown). No other functional sodium channel genes were represented more than $5 \%$. Cardiomyocytes were held at $-120 \mathrm{mV}$, and currents were evoked by depolarizations to $-10 \mathrm{mV}$ every 5 seconds. This infrequent pulsing protocol reveals drug interactions with the resting or open state of the channel and minimizes the effects of UDB, thereby providing a reasonable estimate of the extent to which GS-967 and eleclazine produce $I_{\mathrm{NaP}}$ tonic block. In all experiments, we used the single dose method to test the compounds. As shown in Figure 1, GS-967 or eleclazine at $1 \mu \mathrm{M}$ exhibited limited tonic block of $I_{\mathrm{NaP}}(14 \% \pm 2 \%$ and $6 \% \pm 3 \%$ for GS-967 and eleclazine, respectively; $n=25-27)$. The maximum tonic block observed for GS-967 and eleclazine was $34 \% \pm 3 \%$ and $43 \% \pm 7 \%$ at 10 and $100 \mu \mathrm{M}$, respectively (Fig. 1). Higher concentrations could not be assayed due to the limited solubility of both compounds. To study the pharmacology of $I_{\mathrm{Na}}$ in hiPSC-derived cardiomyocytes, we recorded sodium current from more than 2500 cells (Supplemental Fig. 1).

Figure 1 shows the concentration-response curves for $I_{\mathrm{NaP}}$ tonic block comparing GS-967, eleclazine, ranolazine, lidocaine, and lacosamide. GS-967 and eleclazine exhibited similar $\mathrm{IC}_{50}$ values (1 and $2.5 \mu \mathrm{M}$, respectively) and had limited tonic block efficacy (Table 1). By contrast, ranolazine, lidocaine, and lacosamide exerted stronger maximum tonic block (for the maximal concentration tested) but with lower potency $\left(\mathrm{IC}_{50}=457,1175\right.$, and $1241 \mu \mathrm{M}$, respectively). We concluded that GS-967 and eleclazine exert less tonic block of $I_{\mathrm{NaP}}$ in hiPSC-derived cardiomyocytes compared with ranolazine, lidocaine, and lacosamide.

Use-Dependent Block of $I_{\mathrm{NaP}}$ in hiPSC-Derived Cardiomyocytes. We previously demonstrated that GS967 exerts a strong UDB of $\mathrm{Na}_{\mathrm{V}} 1.5 I_{\mathrm{NaP}}$ in heterologous cells with greater potency than lidocaine and ranolazine (Potet et al., 2016). Others have shown that eleclazine can also exert UDB of Nav1.5 expressed in tsA201 cells (El-Bizri et al., 2018a,b). This motivated us to examine whether GS-967 and eleclazine exert similar effects in human cardiomyocytes.

We compared UDB by GS-967, eleclazine, ranolazine, lidocaine, and lacosamide in hiPSC-derived cardiomyocytes using two different protocols. First, we applied a series of 30 short (20-millisecond) depolarizing pulses (to $-20 \mathrm{mV}$ ) at two frequencies (2 and $10 \mathrm{~Hz}$ ). Second, we used longer (400millisecond) depolarizing pulses $(-20 \mathrm{mV})$ at a low frequency $(2 \mathrm{~Hz})$ to mimic the physiologic cardiac cycle. In the absence of drugs, there was an $\sim 4 \%$ and $\sim 14 \%$ reduction of channel availability (frequency-dependent inactivation) with the short pulse protocol at 2 and $10 \mathrm{~Hz}$, respectively, and an $\sim 23 \%$ reduction with the longer pulse protocol (Supplemental Figs. 
TABLE 1

Compiled $\mathrm{IC}_{50}$ values and Hill coefficients for GS-967, eleclazine, ranolazine, lidocaine, and lacosamide for tonic block and UDB in hiPSC-derived cardiomyocytes

\begin{tabular}{|c|c|c|c|c|c|}
\hline & GS-967 & Eleclazine & Ranolazine & Lidocaine & Lacosamide \\
\hline \multicolumn{6}{|l|}{ Tonic block } \\
\hline $\mathrm{IC}_{50}(\mu \mathrm{M})$ & 1 & 2.5 & 457.8 & 1175 & 1241 \\
\hline $95 \% \mathrm{CI}(\mu \mathrm{M})^{a}$ & $0.47-1119$ & $0.5-5.4$ & $99.7-1100$ & $826-1710$ & $1070-1486$ \\
\hline Hill coefficient & 1 & 1.3 & 0.7 & 3.6 & 0.9 \\
\hline \multicolumn{6}{|l|}{ UDB $2 \mathrm{~Hz}$} \\
\hline $\mathrm{IC}_{50}(\mu \mathrm{M})$ & 0.09 & 0.9 & 8.1 & 156.6 & 249.5 \\
\hline $95 \% \mathrm{CI}(\mu \mathrm{M})$ & $0.07-0.12$ & $0.16-6.31$ & $6.5-10.7$ & $83.8-453.5$ & $158.4-876.6$ \\
\hline Hill coefficient & 2.8 & 1.2 & 1.9 & 1.4 & 1 \\
\hline \multicolumn{6}{|l|}{ UDB $10 \mathrm{~Hz}$} \\
\hline $\mathrm{IC}_{50}(\mu \mathrm{M})$ & 0.07 & 0.6 & 7.8 & 133.5 & 158.5 \\
\hline $95 \% \mathrm{CI}(\mu \mathrm{M})$ & $0.06-0.08$ & $0.28-1007$ & $6.7-9.1$ & $63.9-1161$ & $131.4-212.9$ \\
\hline Hill coefficient & 1.8 & 1 & 2.8 & 0.7 & 1.2 \\
\hline \multicolumn{6}{|c|}{ UDB $2 \mathrm{~Hz}(400 \mathrm{~ms})$} \\
\hline $\mathrm{IC}_{50}(\mu \mathrm{M})$ & 0.05 & 0.4 & 6.5 & 32.6 & 40.6 \\
\hline $95 \% \mathrm{CI}(\mu \mathrm{M})$ & $0.04-0.06$ & $0.28-0.57$ & $5.1-8.2$ & $23.1-49$ & $31.8-51.1$ \\
\hline Hill coefficient & 2.6 & 1.8 & 2.7 & 1.1 & 1.3 \\
\hline
\end{tabular}

95\% CI, 95\% confidence interval.

${ }^{a}$ The confidence intervals for $\mathrm{IC}_{50}$ values were calculated using the asymmetric (likelihood) method and determined from one fit of the pooled data.

2-4). After bath application of drugs, repetitive pulsing was associated with a progressive reduction of $\mathrm{Na}_{\mathrm{V}} 1.5 I_{\mathrm{NaP}}$ consistent with UDB and frequency-dependent inactivation of the channel (Supplemental Figs. 2-4). To quantify the extent of UDB, we normalized the current amplitude measured after drug exposure by the current measured before drug application (Fig. 2A). The potency of each drug at producing UDB was compared by plotting the concentration-response curves (Fig. 2, B-D).

All drugs exhibited limited UDB at $2 \mathrm{~Hz}$ using the 20 millisecond voltage step (Fig. 2B; Supplemental Fig. 2). However, at $10 \mathrm{~Hz}$ and at $2 \mathrm{~Hz}$ using the longer protocol, all drugs showed greater UDB efficacy (Fig. 2, C and D; Supplemental Figs. 3 and 4). Eleclazine and GS-967 exhibited much greater UDB potency than ranolazine, lidocaine, and lacosamide. The order of UDB potency for the five drugs tested was the same as that found for tonic block. The calculated $\mathrm{IC}_{50}$ values for UDB at $10 \mathrm{~Hz}$ were $0.07 \mu \mathrm{M}$ for GS-967, $0.6 \mu \mathrm{M}$ for eleclazine, $7.8 \mu \mathrm{M}$ for ranolazine, $133.5 \mu \mathrm{M}$ for lidocaine, and $158.5 \mu \mathrm{M}$ for lacosamide (Fig. 2C; Table 1). At $10 \mathrm{~Hz}$, eleclazine and GS-967 were 222-fold and 1907-fold more potent, respectively, than lidocaine, one of the best characterized use-dependent sodium channel blockers.

Eleclazine and GS-967 Affect Recovery from Inactivation and Exhibit Moderate Unbinding Kinetics. To explore plausible mechanisms to explain the UDB of $I_{\mathrm{NaP}}$ in hiPSC-derived cardiomyocytes by GS-967 and eleclazine, we first examined recovery from slow inactivation. This was done by utilizing a standard two-pulse protocol consisting of a depolarizing $(-20 \mathrm{mV})$ 1000-millisecond pulse to reach maximum inhibition of peak $I_{\mathrm{Na}}$, followed by a variable duration recovery step to $-120 \mathrm{mV}$ and a final test pulse ( $-20 \mathrm{mV}, 20$ milliseconds) (voltage protocol illustrated in Supplemental Fig. 5). Channel availability after the end of the recovery interval was normalized to initial values and plotted against the recovery time. The time course of recovery was slowed by all the drugs in a concentration-dependent manner (Supplemental Fig. 5). The delayed recovery from inactivation reflects the dissociation of the drug from the channels, which can be quantified by comparing the recovery rate in the absence and presence of the compound (to remove drug-independent inactivation) (Fig. 3). The $\mathrm{K}_{\mathrm{OFF}}$ was then calculated by fitting the dissociation time course. The calculated $\mathrm{K}_{\mathrm{OFF}}$ was 1.6 and 1.5 second $^{-1}$ for GS-967 and eleclazine, respectively, which is similar to lidocaine $\left(1.1\right.$ second $\left.^{-1}\right)$ but an order of magnitude slower than ranolazine $\left(16.2\right.$ second $\left.^{-1}\right)$ and four times more rapid than lacosamide $\left(0.4\right.$ second $\left.^{-1}\right)$ (Fig. 3).

Eleclazine and GS-967 Exhibit Rapid Binding Kinetics. Because UDB can be due to accumulation of channels in a slow inactivated state evoked by prolonged membrane depolarization, we investigated whether kinetic differences in the rate of entry into slow inactivation could account for UDB by GS-967 and eleclazine in hiPSC cardiomyocytes. To assess the onset of slow inactivation, cells were held at $120 \mathrm{mV}$ and then depolarized to $-20 \mathrm{mV}$ for a variable duration (2-1000 milliseconds) followed by a brief recovery pulse ( $-120 \mathrm{mV}$ for 20 milliseconds) and a final 20 -millisecond test pulse to $-20 \mathrm{mV}$ (voltage protocol illustrated in Fig. 4; Supplemental Fig. 6). The proportion of channels entering slow inactivation was estimated by normalizing to initial values (current recorded at P1) the current obtained after the short recovery pulse (current recorded at P2) and plotted against the first pulse duration. To assess the development of inhibition, we normalized the development of slow inactivation in the absence and presence of the compound to remove drug-independent inactivation (Fig. 4, A-D). We could not measure the development of inhibition for ranolazine due to its short dissociation time constant $\left(\tau_{\mathrm{OFF}}=62\right.$ milliseconds, Fig. 3). The brief 20-millisecond recovery pulse to $-120 \mathrm{mV}$ was long enough to allow ranolazine to apparently unbind. The time-dependent inhibition of the $\mathrm{Na}^{+}$currents shown in (Fig. 4, A-D) was fitted with an exponential decay function having time constant $\tau$. The $1 / \tau$ values were then plotted against the drug concentrations and best fitted with a linear function (Fig. 4E). The slope represents the apparent $\mathrm{K}_{\mathrm{ON}}$ of the drug. As shown in Figure 4E, the $\mathrm{K}_{\mathrm{ON}}$ for GS-967, eleclazine, lidocaine, and lacosamide was $25.7,4.6,0.2$, and 0.003 second ${ }^{-1} \mu \mathrm{M}^{-1}$, respectively. The $\mathrm{K}_{\mathrm{ON}}$ for ranolazine was estimated from the $\mathrm{Kd}$ value $(6.5 \mu \mathrm{M}$; equivalent to the 
A
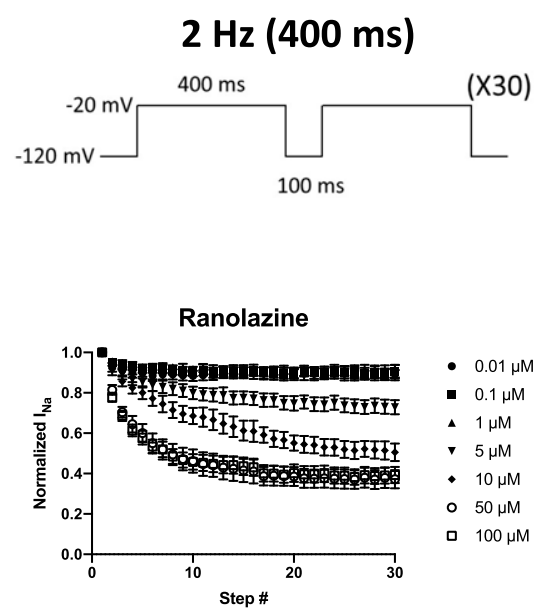

B
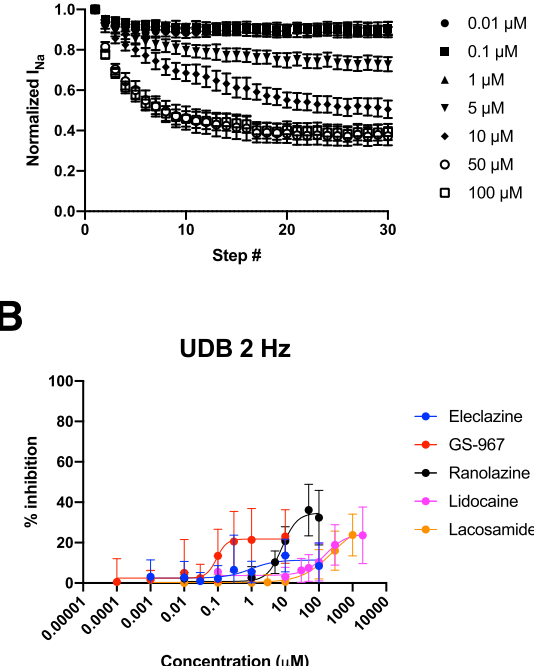

$0.1 \mathrm{~m}$

$1 \mu \mathrm{M}$

- $5 \mu \mathrm{M}$

$100 \mu \mathrm{M}$

Concentration $(\mu \mathrm{M})$
GS-967

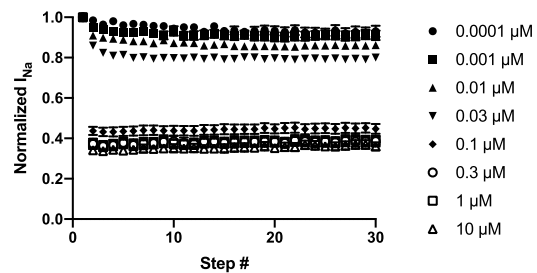

Lidocaine

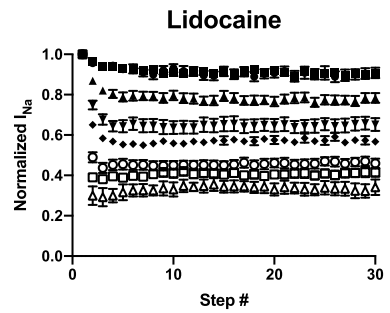

C

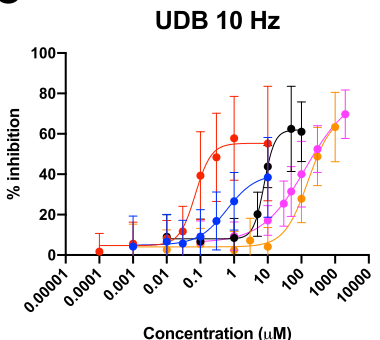

Eleclazine

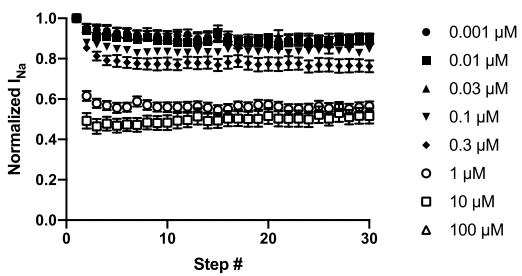

Lacosamide

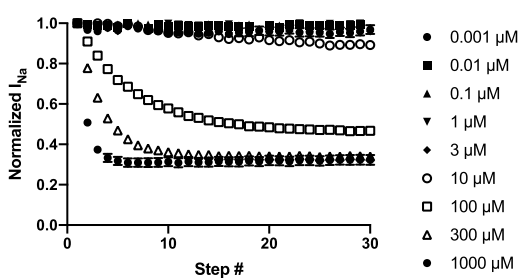

D

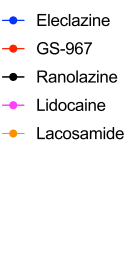

UDB 2 Hz (400 ms)

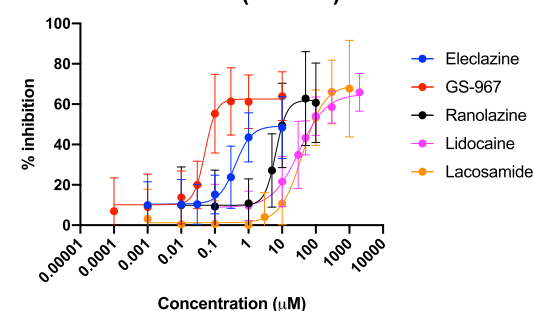

Fig. 2. Concentration-response of $I_{\mathrm{NaP}}$ use-dependent block in hiPSC-derived cardiomyocytes by GS-967, eleclazine, ranolazine, lidocaine, and lacosamide. (A) To examine use-dependent block, hiPSC-derived cardiomyocytes were held at $-120 \mathrm{mV}$ and pulsed to $-20 \mathrm{mV}$ for $400 \mathrm{milliseconds}$ at 2 $\mathrm{Hz}$, with an interpulse potential of $-120 \mathrm{mV}$ (see inset). The peak currents elicited by each pulse were normalized to the peak current of first pulse and plotted against the pulse number. The ratio of current recorded after/before drug was plotted to assess the potency of pure UDB. GS-967: concentrations from 0.0001 to $10 \mu \mathrm{M} ; n=35-67$. Eleclazine: concentrations from 0.0001 to $100 \mu \mathrm{M} ; n=16-29$. Ranolazine concentrations from 0.01 to $100 \mu \mathrm{M} ; n=23-33$. Lidocaine concentrations from 0.1 to $2000 \mu \mathrm{M} ; n=6-27$. Lacosamide concentrations from 0.001 to $1000 \mu \mathrm{M} ; n=41-145$. Concentration-response relationships for use-dependent block were studied using a series of 30 short (20-millisecond) depolarizing pulses $(-20 \mathrm{mV})$ at two different frequencies $(2$ and $10 \mathrm{~Hz})$ or longer physiologic ( 400 -millisecond) depolarizing pulses $(-20 \mathrm{mV})$ at a low frequency $(2 \mathrm{~Hz})$. The \% inhibition at the 30 th pulse was calculated and plotted as a function of drug concentration. (B) Concentration-response relationships for the use-dependent block at $2 \mathrm{~Hz}$ using a 20-millisecond step. $\mathrm{IC}_{50}=0.09,0.9,8.1,156.6$, and $249.5 \mu \mathrm{M}$ for GS-967, eleclazine, ranolazine, lidocaine, and lacosamide, respectively. (C) Concentration-response for use-dependent block at $10 \mathrm{~Hz}$ using a 20-millisecond step. $\mathrm{IC}_{50}=0.07,0.6,7.8,133.5$, and $158.5 \mu \mathrm{M}$ for GS-967, eleclazine, ranolazine, lidocaine, and lacosamide, respectively. (D) Concentration-response for use-dependent block at $2 \mathrm{~Hz}$ using a 400 -millisecond step. IC $50=0.05$, 0.4, 6.5, 32.6, and 40.6 $\mu \mathrm{M}$ for GS-967, eleclazine, ranolazine, lidocaine, and lacosamide, respectively. Data are presented as means \pm S.E.M. in (A) for clarity and means \pm S.D. in (B-D). The $95 \%$ confidence intervals and Hill coefficients are provided in Table 1 . The curves described by the solid lines were fitted by a four-parameter logistic equation.

$\mathrm{K}_{\mathrm{OFF}} / \mathrm{K}_{\mathrm{ON}}$ ratio). This $\mathrm{Kd}$ value is nearly identical to the $\mathrm{IC}_{50}$ value measured in the dose-response curve (Fig. 2D). The estimated $\mathrm{K}_{\mathrm{ON}}$ for ranolazine was 2.5 second $^{-1} \mu \mathrm{M}^{-1}$. Data for all five drugs are summarized in Figure 5. Eleclazine and GS967 exhibit a higher association rate compared with ranolazine, lidocaine, and lacosamide but have rapid dissociation rates similar to lidocaine. Based on this analysis, the greater UDB potency of GS-967 and eleclazine is well correlated with the higher association rates of these two compounds.

\section{Discussion}

In this study, we used hiPSC-derived cardiomyocytes and automated patch clamp recording to elucidate the mechanism of action of two novel sodium channel blockers with demonstrated potent antiarrhythmic effects in various in vitro and in vivo models. Previously, the antiarrhythmic effects of GS967 and eleclazine were attributed to preferential suppression of $I_{\mathrm{NaL}}$ (Belardinelli et al., 2013; Pezhouman et al., 2014; Burashnikov et al., 2015; Fuller et al., 2016; Bacic et al., 2017;
Bossu et al., 2018; El-Bizri et al., 2018a). Here we offer evidence for other potentially important biophysical effects of GS-967 and eleclazine on sodium channels in human cardiomyocytes. We specifically demonstrated that GS-967 and eleclazine exert strong effects on slow inactivation and recovery from inactivation, resulting in a substantial UDB similar to class Ib antiarrhythmic drugs. These revelations may help explain the pharmacological effects of GS-967 and eleclazine in arrhythmia models.

A novel feature of our study is the use of automated planar patch clamp recording of hiPSC-derived cardiomyocytes in a 384-well configuration. Although the use of planar patch clamp to record $\mathrm{Na}^{+}$current in hiPSC-derived cardiomyocytes was described previously (Rajamohan et al., 2016; Li et al., 2019), these reports used much lower throughput platforms capable of recording from only four to eight cells at a time. Our use of the 384-well platform in our study enabled a more robust capability to examine the pharmacological actions of multiple drugs simultaneously. The higher throughput also allowed us to test single drug concentrations per well and to 


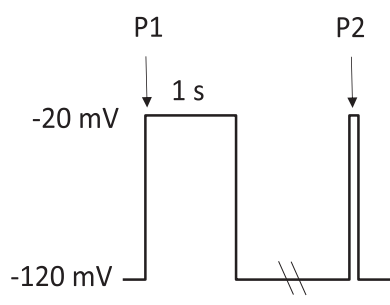

$\Delta \mathrm{t}$

C

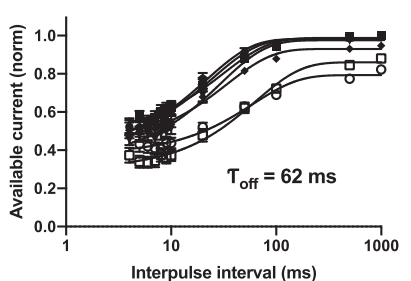

A

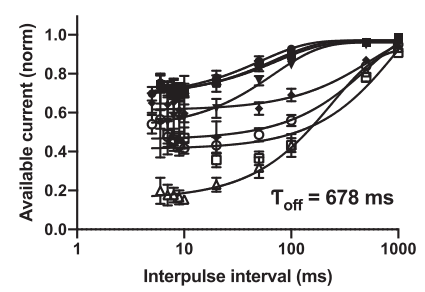

D

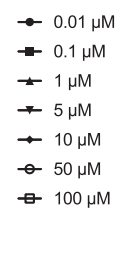

Lidocaine

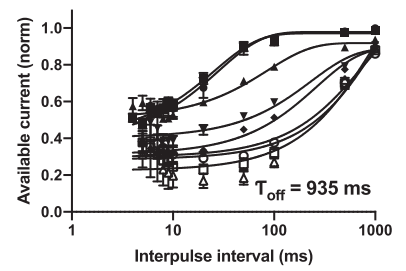

B

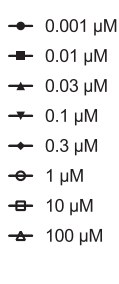

$0.001 \mu \mathrm{M}$

$-0.01 \mu \mathrm{M}$

$\rightarrow 0.3 \mu \mathrm{M}$

$-1 \mu \mathrm{M}$

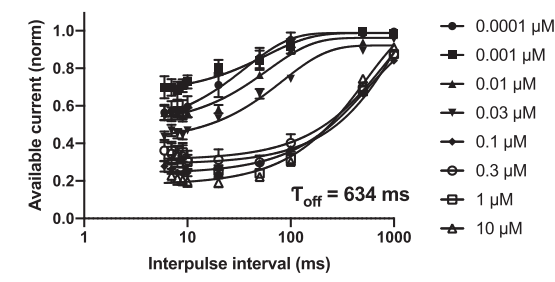

E

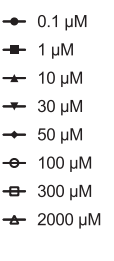

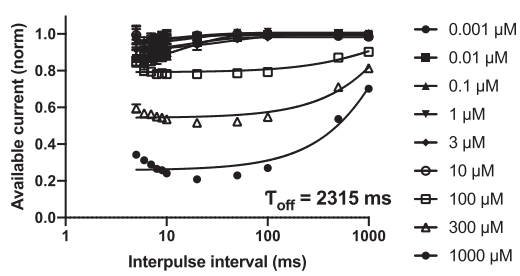

Fig. 3. Recovery from inhibition in hiPSC-derived cardiomyocytes by GS-967, eleclazine, ranolazine, lidocaine, and lacosamide. Recovery from inactivation was studied in hiPSC-derived cardiomyocytes with a standard two-pulse protocol consisting of a depolarizing ( $-20 \mathrm{mV}) 1000-\mathrm{millisecond}$ pulse to engage slow inactivation, followed by a variable duration recovery step to $-120 \mathrm{mV}$ and a final test pulse $(-20 \mathrm{mV}$, $20 \mathrm{milliseconds})$; see inset. Available current at the end of the recovery interval was normalized to initial values and plotted against the recovery time. To calculate the $\mathrm{K}_{\mathrm{OFF}}$, the recovery of drug-bound channels was distinguished from nonbound channels by the ratio ( $\left.\mathrm{I}_{\text {DRUG_REC }} / \mathrm{I}_{\text {DRUG_MAX }}\right) /\left(\mathrm{I}_{\mathrm{CTR} \_ \text {REC }} / \mathrm{I}_{\mathrm{CTR} \_ \text {MAX }}\right.$ ), where $\mathrm{I}_{\mathrm{X} \_ \text {REC }}$ is the proportion of channels recovered in the presence or absence of a drug and $\mathrm{I}_{\mathrm{X} \_\mathrm{MAX}}$ is the maximum current obtained in the presence or absence of drug (El-Bizri et al., 2018a). The recovery time course was fit with a single exponential equation to obtain a recovery time constant, $\tau_{\text {OFF }}$. The exponential curves appear sigmoidal because they were plotted on a log axis. The recovery time constant was averaged for each drug, and the $\mathrm{K}_{\mathrm{OFF}}$ was calculated using $\mathrm{K}_{\mathrm{OFF}}=1 / \tau_{\mathrm{OFF}}$. (A) GS-967: $\mathrm{K}_{\mathrm{OFF}}=1.58$ second $^{-1} ; n=12-32$. (B) Eleclazine: $\mathrm{K}_{\mathrm{OFF}}=1.48$ second $^{-1} ; n=23-24$. (C) Ranolazine: $\mathrm{K}_{\mathrm{OFF}}=16.2$ second ${ }^{-1}$; $n=16-19$. (D) Lidocaine: $\mathrm{K}_{\mathrm{OFF}}=1.1$ second $^{-1} ; n=3-10$. (E) Lacosamide: $\mathrm{K}_{\mathrm{OFF}}=0.4$ second $^{-1} ; n=73-127$. Data represent means $\pm \mathrm{S} . \mathrm{E} . \mathrm{M}$. for clarity.

measure binding and unbinding kinetics for five different drugs. These data demonstrate the feasibility of studying the pharmacological actions of drugs on the native human cardiac sodium current in hiPSC-derived cardiomyocytes.

Unique to our study, we demonstrated that GS-967 and eleclazine exert potent UDB of $I_{\mathrm{NaP}}$ in hiPSC-derived cardiomyocytes. Previously, UDB of GS-967 and eleclazine has not been examined in hiPSC-derived cardiomyocytes (Belardinelli et al., 2013; Bonatti et al., 2014; Alves Bento et al., 2015; Burashnikov et al., 2015; Carneiro et al., 2015; Fuller et al., 2016; Portero et al., 2017; El-Bizri et al., 2018a). Among the sodium channel blockers we tested, GS-967

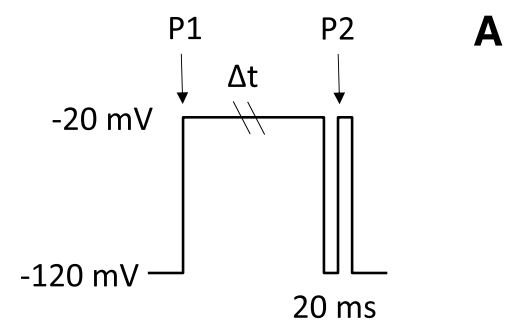

C

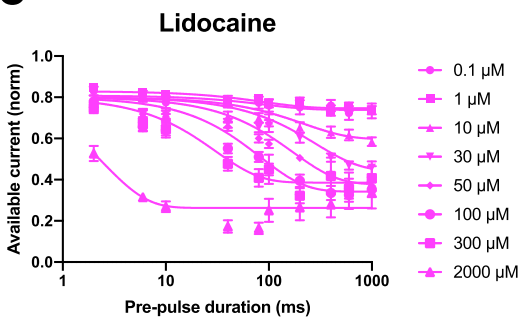

\section{A}

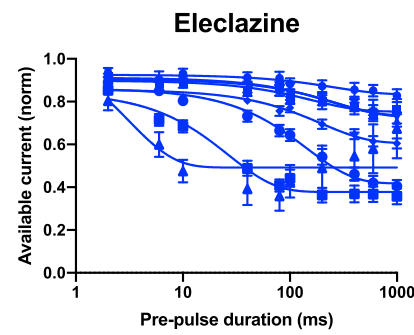

D

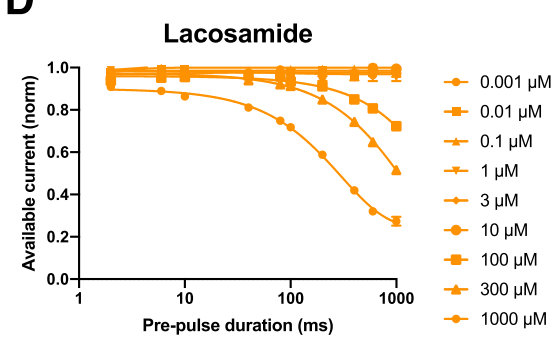

B
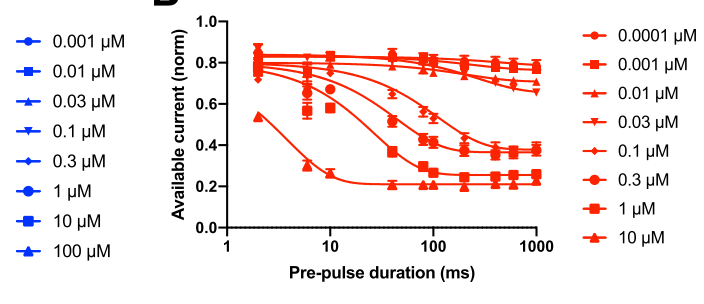

E

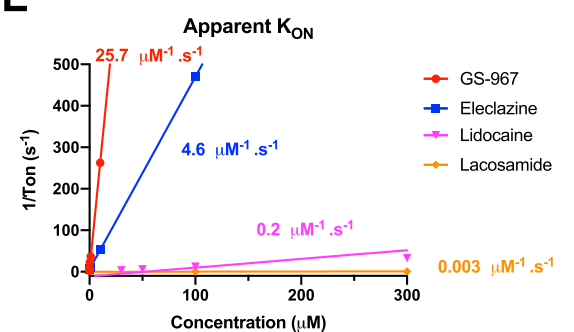

Fig. 4. The apparent inhibition rate $\left(\mathrm{K}_{\mathrm{ON}}\right)$ of GS-967 and eleclazine is more rapid than ranolazine, lidocaine, and lacosamide. The onset of slow inactivation induced by GS-967, eleclazine, ranolazine, lidocaine, and lacosamide was determined in hiPSC-derived cardiomyocytes using the two-pulse protocol illustrated in the inset. Concentration-response curves were plotted using the data collected after a prepulse of 1000 milliseconds. The development of slow inactivation in the presence of compounds was normalized by the values determined in the absence of the compound to remove drugindependent inactivation. The time-dependent inhibition of $I_{\mathrm{NaP}}$ shown for eleclazine (A), GS-967 (B), lidocaine (C), and lacosamide (D) was fitted by an exponential decay function with a time constant $(\tau)$. The exponential curves appear sigmoidal because they were plotted on a log axis. (E) Values for $1 / \tau$ were plotted against the drug concentrations and best fitted by a linear function. The slope represents the apparent $\mathrm{K}_{\mathrm{ON}}$ of the drug. $\mathrm{K}_{\mathrm{ON}} \mathrm{values}$ for GS967, eleclazine, lidocaine, and lacosamide were $25.7,4.6,0.2$, and 0.003 second $^{-1} \mu \mathrm{M}^{-1}$, respectively. Data are presented as means \pm S.E.M. for clarity. 


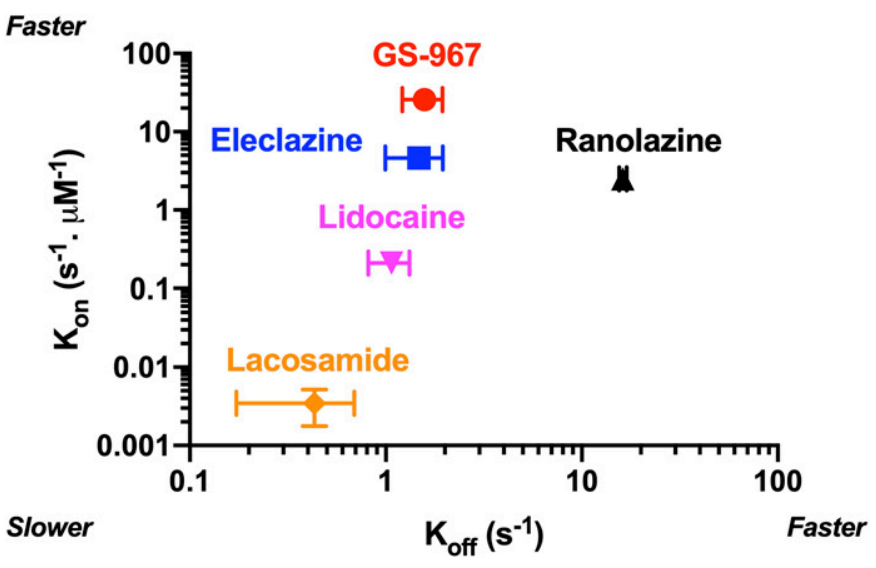

Fig. 5. Relationship between $\mathrm{K}_{\mathrm{ON}}$ and $\mathrm{K}_{\mathrm{OFF}}$ for GS-967, eleclazine, ranolazine, lidocaine, and lacosamide in hiPSC-derived cardiomyocytes. Relationship between the measured $\mathrm{K}_{\mathrm{ON}}$ and the measured $\mathrm{K}_{\mathrm{OFF}}$ for GS967, eleclazine, lidocaine, and lacosamide in hiPSC-derived cardiomyocytes. The $\mathrm{K}_{\mathrm{ON}}$ for ranolazine was estimated as 2.5 second $^{-1} \mu \mathrm{M}^{-1}$ from $\mathrm{Kd}$ $=\mathrm{K}_{\mathrm{OFF}} / \mathrm{K}_{\mathrm{ON}}$. This $\mathrm{Kd}$ value is nearly identical to the $\mathrm{IC}_{50}$ value measured in the dose-response curve (Fig. 2D). The horizontal error bars represent the S.D. for $\mathrm{K}_{\mathrm{OFF}}$. Confidence intervals for $\mathrm{K}_{\mathrm{ON}}$ are smaller than the data symbols except for lacosamide.

exhibited the greatest UDB potency, with $\mathrm{IC}_{50}$ values ranging from 50 to $90 \mathrm{nM}$ depending on stimulation frequency and duration of the voltage steps (Fig. 2). GS-967 and eleclazine exerted UDB that was qualitatively similar to lidocaine (a prototypic use-dependent blocker of sodium channels) but with a greater potency. The greater UDB potency was correlated with the unique on and off rate kinetics for these novel sodium channel blockers. By contrast, the rapid dissociation of ranolazine $\left(\mathrm{K}_{\mathrm{OFF}}\right.$, Figs. 3 and 5$)$ and the slow binding kinetics of lacosamide ( $\mathrm{K}_{\mathrm{ON}}$, Figs. 4 and 5) were both correlated with less potent UDB.

GS-967 and eleclazine were originally demonstrated to exert potent antiarrhythmic effects in rabbit ventricular, canine, and pig myocytes/heart by a proposed mechanism of action involving preferential $I_{\mathrm{NaL}}$ block (Belardinelli et al., 2013; Sicouri et al., 2013; Fuller et al., 2016; Bacic et al., 2017). In a more recent study, despite completely abolishing dofetilideinduced torsades de pointes in a chronic atrioventricular block dog model, GS-967 did not completely suppress early afterdepolarizations (Bossu et al., 2018). The authors concluded that GS-967 predominantly affects the perpetuation, but not the initiation, of arrhythmic events into torsades de pointes and that $I_{\mathrm{NaL}}$ density does not play a critical role in the moderate in vitro antiarrhythmic effect. Because the GS-967 and eleclazine concentrations used in those studies range from 0.2 to $1 \mu \mathrm{M}$, a range sufficient to suppress $I_{\mathrm{NaL}}$ and also to evoke UDB, we speculate that antiarrhythmic effects of these compounds may be due to the combination of $I_{\mathrm{NaL}}$ suppression and UDB preventing the perpetuation of the arrhythmia.

The effects of GS-967 and eleclazine resemble the effects of lidocaine, a class Ib antiarrhythmic drug (Fig. 2). Effective class I antiarrhythmic drugs exhibit a greater efficacy in situations associated with rapid repetitive firing of action potentials (UDB) or prolonged tissue depolarization. Thus, effective arrhythmia suppression depends on the properties of the drug molecule that convey high affinity binding to the receptor on the channel pore when the channel is in the open or inactivated state. Such high affinity binding results in a slowed recovery of the drug-bound channel from inactivation as the cell membrane repolarizes (Ragsdale et al., 1996). In this study, we show that GS-967 and eleclazine, in hiPSC-derived cardiomyocytes, have very high association rates and moderate residence time comparable to lidocaine (Fig. 5). The moderate unbinding kinetics observed for GS-967 and eleclazine would limit peak $I_{\mathrm{Na}}$ inhibition and maintain the conduction velocity (Rajamani et al., 2016). The rapid binding of GS-967 and eleclazine would promote inhibition of late $I_{\mathrm{Na}}$ during phases 2 and 3 of the action potential and exert an antiarrhythmic action in the context of type 3 long QT syndrome (El-Bizri et al., 2018b). This was the rationale for clinical trials of eleclazine for type 3 long QT syndrome (https://clinicaltrials.gov/show/NCT02300558).

In conclusion, we demonstrated the feasibility of using high throughput automated patch clamp recording to examine block of cardiac sodium current by multiple drugs in hiPSCderived cardiomyocytes. We also demonstrated that GS-967 and eleclazine are more potent use-dependent blockers of cardiomyocyte sodium current than the antiarrhythmic drugs lidocaine and ranolazine or the antiepileptic drug lacosamide. We propose that potent UDB contributes to the antiarrhythmic effects of GS-967 and eleclazine.

\section{Acknowledgments}

The authors thank Hui-Hsuan Kuo for her technical assistance.

\section{Authorship Contributions}

Participated in research design: Potet, George.

Conducted experiments: Potet, Egecioglu.

Performed data analysis: Potet.

Wrote or contributed to the writing of the manuscript: Potet, Burridge, George.

\section{References}

Alves Bento AS, Bacic D, Saran Carneiro J, Nearing BD, Fuller H, Justo FA, Rajamani S, Belardinelli L, and Verrier RL (2015) Selective late $\mathrm{I}_{\mathrm{Na}}$ inhibition by GS458967 exerts parallel suppression of catecholamine-induced hemodynamically significant ventricular tachycardia and T-wave alternans in an intact porcine model. Heart Rhythm 12:2508-2514.

Antzelevitch C, Nesterenko V, Shryock JC, Rajamani S, Song Y, and Belardinelli L (2014) The role of late $\mathrm{I}_{\mathrm{Na}}$ in development of cardiac arrhythmias. Handb Exp Pharmacol 221:137-168.

Bacic D, Carneiro JS, Bento AA, Nearing BD, Rajamani S, Belardinelli L, and Verrier RL (2017) Eleclazine, an inhibitor of the cardiac late sodium current, is superior to flecainide in suppressing catecholamine-induced ventricular tachycardia and T-wave alternans in an intact porcine model. Heart Rhythm 14:448-454.

Belardinelli L, Liu G, Smith-Maxwell C, Wang WQ, El-Bizri N, Hirakawa R, Karpinski S, Li CH, Hu L, Li XJ, et al. (2013) A novel, potent, and selective inhibitor of cardiac late sodium current suppresses experimental arrhythmias. J Pharmacol Exp Ther 344:23-32.

Bennett PB, Yazawa K, Makita N, and George AL Jr. (1995) Molecular mechanism for an inherited cardiac arrhythmia. Nature 376:683-685.

Bonatti R, Silva AF, Batatinha JA, Sobrado LF, Machado AD, Varone BB, Nearing BD, Belardinelli L, and Verrier RL (2014) Selective late sodium current blockade with GS-458967 markedly reduces ischemia-induced atrial and ventricular repolarization alternans and ECG heterogeneity. Heart Rhythm 11:1827-1835.

Bossu A, Houtman MJC, Meijborg VMF, Varkevisser R, Beekman HDM, Dunnink A de Bakker JMT, Mollova N, Rajamani S, Belardinelli L, et al. (2018) Selective late sodium current inhibitor GS-458967 suppresses Torsades de Pointes by mostly affecting perpetuation but not initiation of the arrhythmia. $\mathrm{Br} J$ Pharmacol 175: $2470-2482$.

Burashnikov A, Di Diego JM, Goodrow RJ Jr., Belardinelli L, and Antzelevitch C (2015) Atria are more sensitive than ventricles to GS-458967-induced inhibition of late sodium current. J Cardiovasc Pharmacol Ther 20:501-508.

Burridge PW, Holmstrom A, and Wu JC (2015) Chemically defined culture and cardiomyocyte differentiation of human pluripotent stem cells. Curr Protoc Hum Genet 87:21.3.1-21.3.15.

Burridge PW, Li YF, Matsa E, Wu H, Ong SG, Sharma A, Holmström A, Chang AC, Coronado MJ, Ebert AD, et al. (2016) Human induced pluripotent stem cell-derived cardiomyocytes recapitulate the predilection of breast cancer patients to doxorubicin-induced cardiotoxicity. Nat Med 22:547-556.

Burridge PW, Matsa E, Shukla P, Lin ZC, Churko JM, Ebert AD, Lan F, Diecke S, Huber B, Mordwinkin NM, et al. (2014) Chemically defined generation of human cardiomyocytes. Nat Methods 11:855-860.

Carneiro JS, Bento AS, Bacic D, Nearing BD, Rajamani S, Belardinelli L, and Verrier RL (2015) The selective cardiac late sodium current inhibitor GS-458967 
suppresses autonomically triggered atrial fibrillation in an intact porcine model. $J$ Cardiovasc Electrophysiol 26:1364-1369.

El-Bizri N, Li CH, Liu GX, Rajamani S, and Belardinelli L (2018a) Selective inhibition of physiological late $\mathrm{Na}+$ current stabilizes ventricular repolarization. Am $J$ Physiol Heart Circ Physiol 314:H236-H245.

El-Bizri N, Xie C, Liu L, Limberis J, Krause M, Hirakawa R, Nguyen S, Tabuena DR, Belardinelli L, and Kahlig KM (2018b) Eleclazine exhibits enhanced selec tivity for long QT syndrome type 3-associated late $\mathrm{Na}+$ current. Heart Rhythm 15:277-286.

Errington AC, Stöhr T, Heers C, and Lees G (2008) The investigational anticonvulsant lacosamide selectively enhances slow inactivation of voltage-gated sodium channels. Mol Pharmacol 73:157-169.

Fuller H, Justo F, Nearing BD, Kahlig KM, Rajamani S, Belardinelli L, and Verrier RL (2016) Eleclazine, a new selective cardiac late sodium current inhibitor, confers concurrent protection against autonomically induced atrial premature beats, repolarization alternans and heterogeneity, and atrial fibrillation in an intact porcine model. Heart Rhythm 13:1679-1686.

Gupta T, Khera S, Kolte D, Aronow WS, and Iwai S (2015) Antiarrhythmic properties of ranolazine: a review of the current evidence. Int $J$ Cardiol 187:66-74.

Herzog RI, Liu C, Waxman SG, and Cummins TR (2003) Calmodulin binds to the C terminus of sodium channels $\mathrm{Na}_{\mathrm{v}} 1.4$ and $\mathrm{Na}_{\mathrm{v}} 1.6$ and differentially modulates their functional properties. $J$ Neurosci 23:8261-8270.

Hodgkin AL and Huxley AF (1952) A quantitative description of membrane current and its application to conduction and excitation in nerve. J Physiol 117:500-544.

Kahlig KM, Hirakawa R, Liu L, George AL Jr., Belardinelli L, and Rajamani S (2014) Ranolazine reduces neuronal excitability by interacting with inactivated states of brain sodium channels. Mol Pharmacol 85:162-174.

Koltun DO, Parkhill EQ, Elzein E, Kobayashi T, Notte GT, Kalla R, Jiang RH, Li X, Perry TD, Avila B, et al. (2016) Discovery of triazolopyridine GS-458967, a late sodium current inhibitor (Late $\mathrm{I}_{\mathrm{Na}} \mathrm{i}$ ) of the cardiac Nav 1.5 channel with improved efficacy and potency relative to ranolazine. Bioorg Med Chem Lett 26:3202-3206.

Le Grand B, Vie B, Talmant JM, Corabouf E, and John GW (1995) Alleviation of contractile dysfunction in ischemic hearts by slowly inactivating $\mathrm{Na}^{+}$current blockers. Am J Physiol 269:H533-H540.

Li W, Luo X, Ulbricht Y, Wagner M, Piorkowski C, El-Armouche A, and Guan K (2019) Establishment of an automated patch-clamp platform for electrophysiological and pharmacological evaluation of hiPSC-CMs. Stem Cell Res (Amst) $\mathbf{4 1}$ 101662.

Pezhouman A, Madahian S, Stepanyan H, Ghukasyan H, Qu Z, Belardinelli L, and Karagueuzian HS (2014) Selective inhibition of late sodium current suppresses ventricular tachycardia and fibrillation in intact rat hearts. Heart Rhythm 11 492-501.
Pignier C, Rougier JS, Vié B, Culié C, Verscheure Y, Vacher B, Abriel H, and Le Grand B (2010) Selective inhibition of persistent sodium current by F 15845 prevents ischaemia-induced arrhythmias. Br J Pharmacol 161:79-91.

Portero V, Casini S, Hoekstra M, Verkerk AO, Mengarelli I, Belardinelli L, Rajamani S, Wilde AAM, Bezzina CR, Veldkamp MW, et al. (2017) Anti-arrhythmic potential of the late sodium current inhibitor GS-458967 in murine Scn5a-1798insD ${ }^{+/}$and human SCN5A-1795insD ${ }^{+/-}$iPSC-derived cardiomyocytes. Cardiovasc Res 113: 829-838.

Potet F, Vanoye CG, and George AL Jr. (2016) Use-dependent block of human cardiac sodium channels by GS967. Mol Pharmacol 90:52-60.

Ragsdale DS, McPhee JC, Scheuer T, and Catterall WA (1996) Common molecular determinants of local anesthetic, antiarrhythmic, and anticonvulsant block of voltage-gated $\mathrm{Na}^{+}$channels. Proc Natl Acad Sci USA 93:9270-9275.

Rajamani S, Liu G, El-Bizri N, Guo D, Li C, Chen XL, Kahlig KM, Mollova N, Elzein $\mathrm{E}$, Zablocki J, et al. (2016) The novel late $\mathrm{Na}+$ current inhibitor, GS-6615 (eleclazine) and its anti-arrhythmic effects in rabbit isolated heart preparations. $\mathrm{Br}$ $J$ Pharmacol 173:3088-3098.

Rajamohan D, Kalra S, Duc Hoang M, George V, Staniforth A, Russell H, Yang X, and Denning C (2016) Automated electrophysiological and pharmacological evaluation of human pluripotent stem cell-derived cardiomyocytes. Stem Cells Dev 25: 439-452.

Ruan Y, Liu N, and Priori SG (2009) Sodium channel mutations and arrhythmias. Nat Rev Cardiol 6:337-348.

Sicouri S, Belardinelli L, and Antzelevitch C (2013) Antiarrhythmic effects of the highly selective late sodium channel current blocker GS-458967. Heart Rhythm 10: 1036-1043.

Song Y, Shryock JC, Wagner S, Maier LS, and Belardinelli L (2006) Blocking late sodium current reduces hydrogen peroxide-induced arrhythmogenic activity and contractile dysfunction. J Pharmacol Exp Ther 318:214-222.

Strichartz G, Rando T, and Wang GK (1987) An integrated view of the molecular toxinology of sodium channel gating in excitable cells. Annu Rev Neurosci 10: 237-267.

Szél T, Koncz I, Jost N, Baczkó I, Husti Z, Virág L, Bussek A, Wettwer E, Ravens U, Papp JG, et al. (2011) Class I/B antiarrhythmic property of ranolazine, a novel antianginal agent, in dog and human cardiac preparations. Eur J Pharmacol 662: $31-39$.

Address correspondence to: Alfred L. George, Jr., Department of Pharmacology, Northwestern University Feinberg School of Medicine, Searle 8-510, 320 East Superior St, Chicago, IL 60611. E-mail: al.george@northwestern.edu 